\title{
Clinical significance of miR-181a in patients with neonatal sepsis and its regulatory role in the lipopolysaccharide-induced inflammatory response
}

\author{
GUOZHI LIU*, WEI LIU* and JIE GUO \\ Department of Neonatology, Yidu Central Hospital of Weifang, Weifang, Shandong 262500, P.R. China
}

Received April 29, 2019; Accepted October 30, 2019

DOI: $10.3892 /$ etm.2020.8408

\begin{abstract}
Neonatal sepsis (NS) poses a serious threat to the health of neonates worldwide. The present study aimed to investigate the diagnostic value of microRNA (miR)-181a in patients with NS and the regulatory role of miR-181a in lipopolysaccharide (LPS)-induced inflammation. A total of 102 neonates with NS and 50 neonates without sepsis were enrolled in the present study. The serum levels of miR-181a were estimated using reverse transcription-quantitative PCR. Receiver operating characteristic (ROC) analysis was performed to evaluate the diagnostic value of miR-181a for NS. The effect of miR-181a on the expression of Toll-like receptor (TLR)4 was assessed after modification of the expression of miR-181a in monocytes isolated from the blood of neonates in vitro. An ELISA was used to measure the concentration of inflammatory cytokines tumor necrosis factor (TNF)- $\alpha$ and interleukin (IL)- 8 in the supernatant of monocytes. The serum levels of miR-181a were decreased in patients with NS compared with those in the controls. The area under the ROC curve of miR-181a was 0.893 with a sensitivity of $83.3 \%$ and a specificity of $84.0 \%$. LPS stimulation in monocytes also led to a decrease in the expression of miR-181a. TLR4 was proven to be a direct target gene of miR-181a, according to the results of a luciferase reporter assay, and overexpression of miR-181a suppressed TLR4 expression in monocytes. Regarding LPS-induced inflammation, it was revealed that the upregulated levels of TNF- $\alpha$ and IL- 8 induced by LPS were reduced by overexpression of miR-181a in monocytes. In conclusion, decreased serum levels of miR-181a may serve as a diagnostic biomarker in patients with NS and overexpression of miR-181a inhibits the LPS-induced inflammatory response
\end{abstract}

Correspondence to: Dr Guozhi Liu, Department of Neonatology, Yidu Central Hospital of Weifang, 4138 South Linglongshan Road, Weifang, Shandong 262500, P.R. China

E-mail: changshoulmi1@163.com

${ }^{*}$ Contributed equally

Key words: microRNA-181a, neonatal sepsis, diagnosis, inflammatory response, monocyte at least partially by targeting TLR4. Aberrant miR-181a may be a non-invasive biomarker for NS patients, and provide a novel insight into the pathologic mechanisms of action behind the development of NS.

\section{Introduction}

Sepsis is a serious syndrome that is induced by infections and characterized by a systemic inflammatory response (1). Neonatal sepsis (NS) is sepsis that occurs in infants within 28 days of age and is mainly caused by bacterial infection (2). Approximately 1 million infants die from NS every year worldwide, which therefore represents a global health burden (3). However, current strategies for the diagnosis of NS remain limited. Bacterial culture is considered the gold standard for the diagnosis of NS, but it takes 1 to 2 days to obtain the examination results with low sensitivity (4). In addition, several biological markers with high sensitivity have been identified, including interleukins (ILs), C-reactive protein (CRP), micro-erythrocyte sedimentation rate and procalcitonin (PCT) (5-7). However, the clinical application of these indicators is limited due to their poor specificity. Thus, novel diagnostic biomarkers with high sensitivity and specificity are urgently required for the early diagnosis of NS.

MicroRNAs (miRNAs) are small non-coding RNAs that may be easily detected from blood samples (8). It is generally accepted that miRNAs regulate gene expression by directly binding to the 3'-untranslated region (3'-UTR) of target messenger RNA (mRNA), leading to mRNA degradation or suppression of subsequent translation (9). Furthermore, pivotal roles of miRNAs have been demonstrated in a number of biological processes, including cell proliferation, differentiation, cell cycle and cell apoptosis (10). Emerging studies have reported differentially expressed miRNAs in different types of human diseases (11-13). The clinical significance of the aberrant expression of miRNAs has attracted increasing attention due to their high diagnostic and prognostic values $(14,15)$. Downregulated expression of microRNA-181a (miR-181a) has been identified in NS patients by Chen et al (16). A study by He et al (17) demonstrated that miR-181a improved immune thrombocytopenia by regulating Toll-like receptor 4 (TLR4), a key molecule in the innate immune system and the development of NS (18). However, the clinical and biological roles of miR-181a in NS have remained to be fully elucidated. 
To improve the diagnosis of NS, the present study sought to compare the serum levels of miR-181a between NS patients and healthy newborns and explore the diagnostic value of miR-181a. Additionally, the effect of miR-181a on the lipopolysaccharide (LPS)-induced inflammatory response was further analyzed in primary monocytes.

\section{Materials and methods}

Patients and blood sample collection. The experimental protocols were approved by the Ethics Committee of Yidu Central Hospital of Weifang Hospital (Weifang, China) and written informed consent was obtained from the families of the patients. Blood samples were collected from 102 patients with NS at the time of initial laboratory evaluation at the Yidu Central Hospital of Weifang (Weifang, China) between May 2014 and April 2018, and stored at $-80^{\circ} \mathrm{C}$ for further analysis. Furthermore, 50 neonates without any symptoms and signs of sepsis, who underwent routine consultation or vaccination at an outpatient neonatal clinic and were diagnosed with respiratory infection or pneumonia were included in the present study as a control group. The diagnosis of NS was determined based on the criteria established at the 2003 Kunming Neonatal Sepsis Definitions Conference (19); it mainly relies on the clinical manifestations and the detection of blood pathogens. Staphylococcus and Escherichia coli were the most common types among all of the detected bacteria. The clinicopathological characteristics of the participants are listed in Table I.

$R N A$ extraction and reverse transcription-quantitative $P C R$ $(R T-q P C R)$. The collected blood was centrifuged to isolate the serum samples. Total RNA from the serum, including miRNAs, was extracted using TRIzol reagent (Invitrogen; Thermo Fisher Scientific, Inc.) according to the manufacturer's protocol. A NanoDrop 2000 (Thermo Fisher Scientific, Inc.) was used to evaluate the purity and concentration of the RNA. Single-stranded complementary DNA was synthesized from the RNA using the PrimeScript RT reagent kit (Takara Bio, Inc.) and stored at $-20^{\circ} \mathrm{C}$ for subsequent qPCR. The serum levels of miR-181a and mRNA of TLR4 were determined using qPCR, which was performed using a SYBR Green I Master Mix kit (Invitrogen; Thermo Fisher Scientific, Inc.) and a 7300 Real-Time PCR System (Applied Biosystems; Thermo Fisher Scientific, Inc.). The reaction conditions are as follows: miR-181a $95^{\circ} \mathrm{C}$ for $10 \mathrm{~min}, 40$ cycles of 95 es of $\mathrm{m} 0 \mathrm{sec}, 60^{\circ} \mathrm{C}$ for $20 \mathrm{sec}, 72^{\circ} \mathrm{C}$ for $15 \mathrm{sec}$; TLR $495^{\circ} \mathrm{C}$ for $10 \mathrm{~min}, 40$ cycles of $95^{\circ} \mathrm{C}$ for $30 \mathrm{sec}, 58^{\circ} \mathrm{C}$ for $30 \mathrm{sec}, 72^{\circ} \mathrm{C}$ for $20 \mathrm{sec}$. U6 and GAPDH were respectively used as the internal control gene for miR-181a and TLR4. The final expression value was calculated using the $2^{-\Delta \Delta \mathrm{Cq}}$ method (20) and normalized to U6 or GAPDH. The sequences of the primers used in the present study were as follows: miR-181a forward, 5'-GCCGAGAAC AUUCAACGCUGU-3' and reverse, 5'-CTCAACTGGTGT CGTGGA-3'; TLR4 forward, 5'-CAGAGTTGCTTTCAA TGGCATC-3' and reverse, 5'-AGACTGTAATCAAGAACC TGGAGG-3'; U6 forward, 5'-CTCGCTTCGGCAGCACA-3' and reverse, 5'-AACGCTTCACGAATTTGCGT-3'. GAPDH forward, 5'-AGAAGGCTGGGGCTCATTTG-3' and reverse, 5'-AGGGGCCATCCACAGTCTTC-3'.
Cell culture and stimulation conditions. Blood samples collected from the patients with NS were settled by addition of $4.5 \%$ dextran 500 (1:5; Amersham Biosciences) and the leukocytes were separated from the red blood cells. Monocytes were isolated using density gradient centrifugation with FicollPaque (Amersham Pharmacia, Biotech AB) as previously described (21), and the purity of the cells was confirmed to be $>95 \%$ by flow cytometry based on detection of the specific cell markers CD14 and CD45. The extracted monocytes were cultured in RPMI-1640 medium (Gibco; Thermo Fisher Scientific, Inc.) supplemented with $10 \%$ fetal bovine serum (Gibco; Thermo Fisher Scientific, Inc.) in a humidified atmosphere with $5 \% \mathrm{CO}_{2}$ at $37^{\circ} \mathrm{C}$. To explore the effects of miR-181a on LPS-induced inflammation, the monocytes were stimulated using $100 \mathrm{ng} / \mathrm{ml}$ LPS (Sigma-Aldrich; Merck $\mathrm{KGaA}$ ) for $4 \mathrm{~h}$.

Cell transfection. Monocytes were seeded into 48-well plates and transfected with miR-181a mimics, miR-181a inhibitor and miR-negative control (miR-NC) (GenePharma) using Lipofectamine 2000 (Thermo Fisher Scientific, Inc.) according to the manufacturer's protocols. The sequences of the vectors were as follows: miR-181a mimics, 5'-AACAUUCAACGC UGUCGGUGAGU-3'; miR-181a inhibitor, 5'-ACUCACCGA CAGCGUUGAAUGUU-3'; miR-NC, 5'-CAGUACUUUUGU GUAGUACAA-3'.

Luciferase reporter assay. In a bioinformatics analysis using TargetScan (http://www.targetscan.org/vert_72/), a complementary sequence of miR-181a was identified in the 3'-UTR of TLR4. To verify whether there was a direct interaction between miR-181a and TLR4, a luciferase reporter assay was performed in the present study. The wild-type (WT) or mutant-type (MT) 3'-UTR was cloned into the pGL3 basic vector (Promega Corp.) to obtain pLUC-WT-TLR4 or pLUC-MT-TLR4, respectively. miR-181a mimics, miR-181a inhibitor or miR-NC were co-transfected into the isolated monocytes with pLUC-WT-TLR4 or pLUC-MT-TLR4 using Lipofectamine 2000 (Thermo Fisher Scientific, Inc.). A Dual-Luciferase Reporter Assay System (Promega Corp.) was used to measure the luciferase activity in the different groups.

ELISA. The concentration of the inflammatory cytokines tumor necrosis factor (TNF)- $\alpha$ and IL- 8 in the culture supernatant of the monocytes was estimated using ELISA, which was performed using a TNF- $\alpha$ ELISA kit (cat. no. 550610; BD Biosciences) and an IL-8 ELISA kit (cat. no. 550999; BD Biosciences) according to the manufacturer's protocol. The optical density at $450 \mathrm{~nm}$ was read by using a microplate reader (Bio-Rad Laboratories, Inc.).

Statistical analysis. All statistical analyses were performed by using SPSS 18.0 software (SPSS Inc.) and GraphPad Prism 5.0 software (GraphPad Software, Inc., USA). Values are expressed as the mean \pm standard deviation and compared with Student's t-test, the $\chi^{2}$ test or one-way analysis of variance followed by Tukey's multiple-comparisons test. A receiver operating characteristic (ROC) curve was drawn to evaluate the diagnostic value of miR-181a regarding NS. $\mathrm{P}<0.05$ was considered to indicate statistical significance. 
Table I. Clinicopathological characteristics of the NS patients and the controls.

\begin{tabular}{lccr}
\hline Characteristic & Control $(\mathrm{n}=50)$ & NS (n=102) & P-value \\
\hline Age (days) & $11.74 \pm 4.41$ & $11.52 \pm 4.01$ & 0.758 \\
Sex (male/female) & $28 / 22$ & $53 / 49$ & 0.730 \\
Body weight (g) & $3485.18 \pm 308.79$ & $3472.77 \pm 299.91$ & 0.813 \\
CRP (mg/l) & $10.89 \pm 4.74$ & $12.46 \pm 5.86$ & 0.099 \\
WBC $\left(\mathrm{x} 10^{9} / \mathrm{l}\right)$ & $10.62 \pm 4.92$ & $11.59 \pm 5.77$ & 0.307 \\
PCT $(\mathrm{ng} / \mathrm{ml})$ & $1.60 \pm 0.75$ & $4.76 \pm 2.72$ & 0.001
\end{tabular}

NS, neonatal sepsis; CRP, C-reactive protein; WBC, white blood cells; PCT, procalcitionin.

\section{Results}

Clinicopathological characteristics of the patients with NS and the controls. A total of 102 patients with NS and 50 controls were included in the present study. The clinicopathological characteristics, including age, sex, body weight, concentration of CRP and PCT, as well as white blood cell (WBC) count, were summarized in Table I. The control group included 28 males and 22 females with the age of $11.74 \pm 4.41$ days, and 53 males and 49 females were included in the NS patients with an average of $11.52 \pm 4.01$ days. There were no significant differences between the NS cases and controls in terms of age, sex, body weight, $\mathrm{CRP}$ and $\mathrm{WBC}$ count (all $\mathrm{P}>0.05$ ), while a higher PCT was observed in the patients with NS compared with that in the controls $(\mathrm{P}=0.001)$.

Serum miR-181a is downregulated in patients with NS. To investigate the role of miR-181a in NS, the serum levels of miR-181a in the NS patients were measured using RT-qPCR. As presented in Fig. 1, the relative serum levels of miR-181a were significantly downregulated in patients with NS compared with those in the controls $(\mathrm{P}<0.01)$.

Diagnostic value of miR-181a in patients with NS. Given the dysregulation of miR-181a in the serum of patients with NS, its clinical significance in the diagnosis of NS was assessed in the present study. A ROC curve for PCT was first constructed based on the established diagnostic value of PCT in NS and its significantly different concentration in the present NS group compared with that in the controls. As presented in Fig. 2A, the area under the curve (AUC) for PCT was 0.874 with a sensitivity of $75.5 \%$ and a specificity of $98.0 \%$ at a cutoff value of 0.980 . The ROC curve for the levels of miR-181a is presented in Fig. 2B, with an AUC of 0.893 , and a sensitivity and specificity of 83.3 and $84.0 \%$, respectively, at a cutoff value of 0.625 .

Expression of miR-181a in LPS-treated monocytes. To investigate the functional role of miR-181a in LPS-induced inflammation, the expression of miR-181a in monocytes treated with LPS was measured. As presented in Fig. 3, the expression of miR-181a was obviously decreased in the monocytes after LPS stimulation compared with that in the untreated cells $(\mathrm{P}<0.01)$.

miR-181a directly regulates the expression of TLR4. TLR4 is a key molecule in the innate immune system and the development

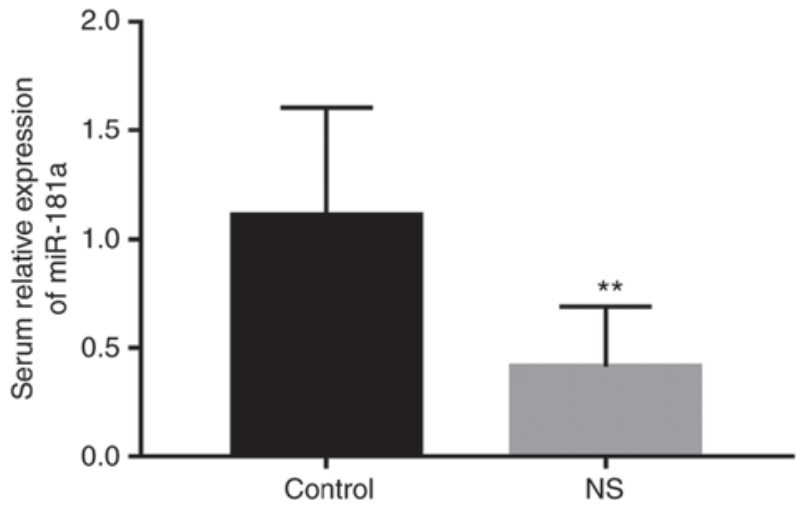

Figure 1. Serum levels of miR-181a in NS patients and controls. The expression of miR-181a was lower in the NS patients than in the controls. ${ }^{* *} \mathrm{P}<0.01$ vs. Control. NS, neonatal sepsis; miR, microRNA.

of NS. TLR4 has been reported to be a direct target of miR-181a in immune thrombocytopenia $(17,18)$. The present study focused on the association between these two molecules in the monocytes. Following transfection with miR-181a mimics or miR-181a inhibitor, the expression of miR-181a was significantly increased or decreased, respectively, as confirmed by RT-qPCR (all $\mathrm{P}<0.01$, Fig. 4A). After the in vitro modification of miR-181a levels, the LPS-induced elevated expression of TLR4 was indicated to be significantly suppressed in the cells with overexpression of miR-181a, whereas it was significantly enhanced in the cells with knockdown of miR-181a (all $\mathrm{P}<0.05$, Fig. 4B), indicating that miR-181a in LPS-treated monocytes led to inhibition of TLR4. In order to further confirm the direct interaction between miR-181a and TLR4, a luciferase reporter assay was performed. A complementary sequence of miR-181a was identified in the 3'-UTR of TLR4 (Fig. 4C). After co-transfection of the reporter vector containing the 3'-UTR sequence of TLR4 and miR-181a mimics or inhibitor, it was observed that the relative luciferase activity in the WT-TLR4 group was markedly decreased in the presence of miR-181a mimics but was increased in the presence of miR-181a inhibitor (all $\mathrm{P}<0$.05, Fig. 4D). However, no significant changes in luciferase activity were observed in the MT-TLR4 groups.

Effects of miR-181a on the levels of pro-inflammatory cytokines in monocytes. The effects of miR-181a on inflammatory cytokines were then investigated to demonstrate the 
A

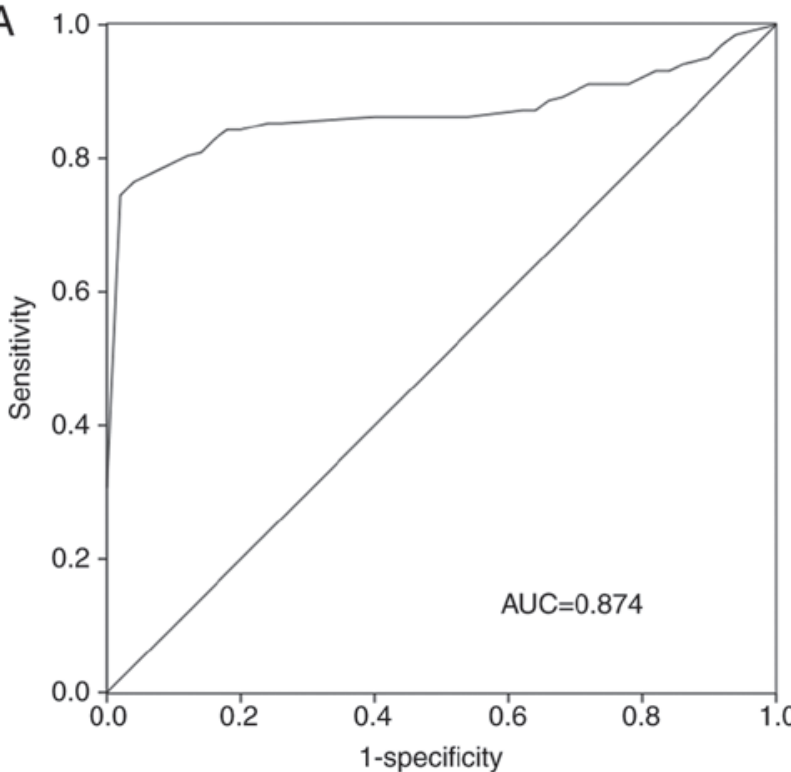

B

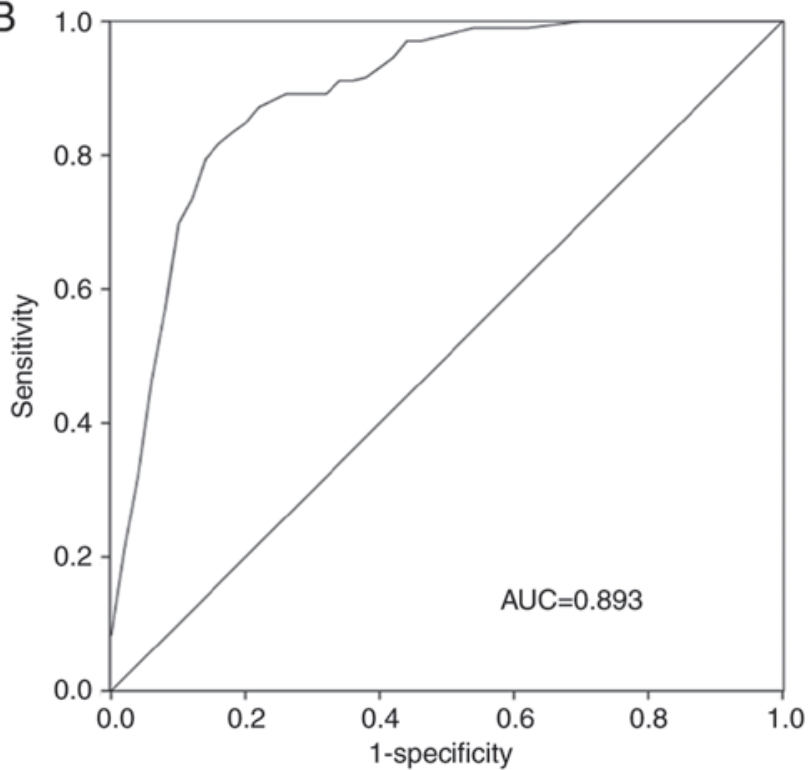

Figure 2. Receiver operating characteristic curves for NS patients based on PCT concentration and miR-181a expression. (A) The AUC of PCT was 0.874 and (B) the AUC of miR-181a expression was 0.893. NS, neonatal sepsis; miR, microRNA; AUC, area under the curve; PCT, procalcitonin.

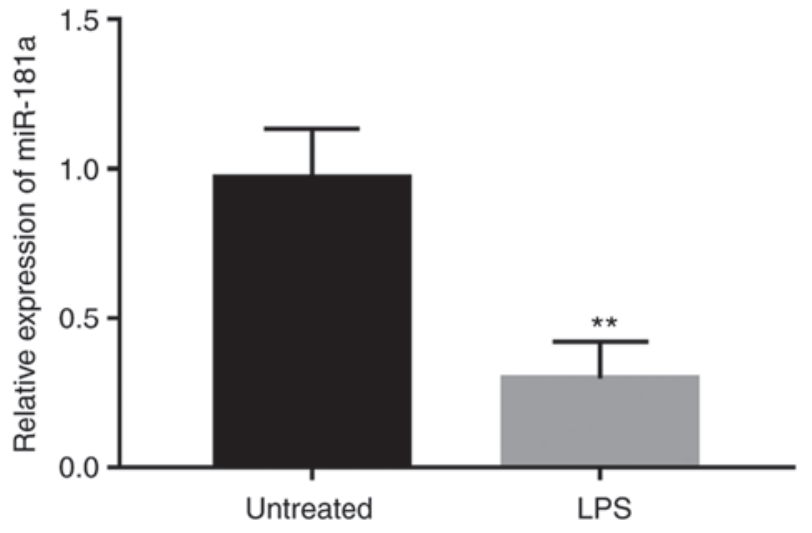

Figure 3. Relative expression of miR-181a in the LPS-treated monocytes. LPS stimulation in the primary monocytes led to a decrease in the expression of miR-181a. ${ }^{* *} \mathrm{P} 0.01$ vs. Untreated. miR, microRNA; LPS, lipopolysaccharide.

regulatory role of miR-181a on inflammation in monocytes. As presented in Fig. 5, the concentration of TNF- $\alpha$ and IL-8 was increased after LPS stimulation (all $\mathrm{P}<0.05$ ). Following modification of miR-181a levels in the monocytes, it was observed that overexpression of miR-181a resulted in decreased levels of TNF- $\alpha$ and IL-8, while inhibition of miR-181a led to increased concentrations of these two cytokines in the presence of LPS (all $\mathrm{P}<0.05)$.

\section{Discussion}

The present study focused on the expression and clinical significance of miR-181a in patients with NS and explored the effects of miR-181a on LPS-induced inflammation in monocytes. RT-qPCR indicated that the serum levels of miR-181a were significantly downregulated in patients with NS compared with those in the controls, which may be of diagnostic value with considerable sensitivity and specificity. In the monocytes extracted from the serum of patients with NS, the expression of miR-181a was also downregulated after LPS stimulation. TLR4 has been previously reported to be a target gene of miR-181a in immune thrombocytopenia (17), and in the present study, it was demonstrated that miR-181a directly inhibits the expression of TLR4 in monocytes. Furthermore, overexpression of miR-181a led to inhibition of LPS-induced inflammation, as evidenced by the decreased TNF- $\alpha$ and IL- 8 concentrations.

Numerous studies have indicated the pivotal roles of miRNAs in the initiation and development of various human diseases, including malignancies (22), metabolic diseases (23) and cardiovascular diseases (24). In sepsis, there are also functional miRNAs that are linked to the progression of the disease by the regulation of inflammatory response, such as miR-150 (25) and miR-27a (26), e.g. miR-375 (25) and miR-25 (26). In addition, certain miRNAs with ectopic expression patterns have critical roles in the pathogenesis of NS by regulating the inflammatory response. For instance, miR-15a/16 has been reported to be upregulated in serum samples of patients with NS and may be involved in the inflammatory response in this disease (18). The expression of miR-132 and miR-223 was demonstrated to be downregulated in patients with NS compared with that in healthy infants and was associated with the expression of immune-associated genes involved in the TLR signaling pathway (27). In the present study, NS patients were recruited to estimate the expression of miR-181a. This study enrolled neonates with respiratory infection or pneumonia as controls but did not include healthy neonates. Firstly, blood samples were difficult to obtain from healthy individuals for ethical considerations; however, neonates with infections, but not sepsis, already underwent blood collection and examination. Thus, their blood samples were available with the approval from the families. Additionally, infections in neonates with pneumonia/respiratory tract infection contribute to the occurrence of NS (28). 

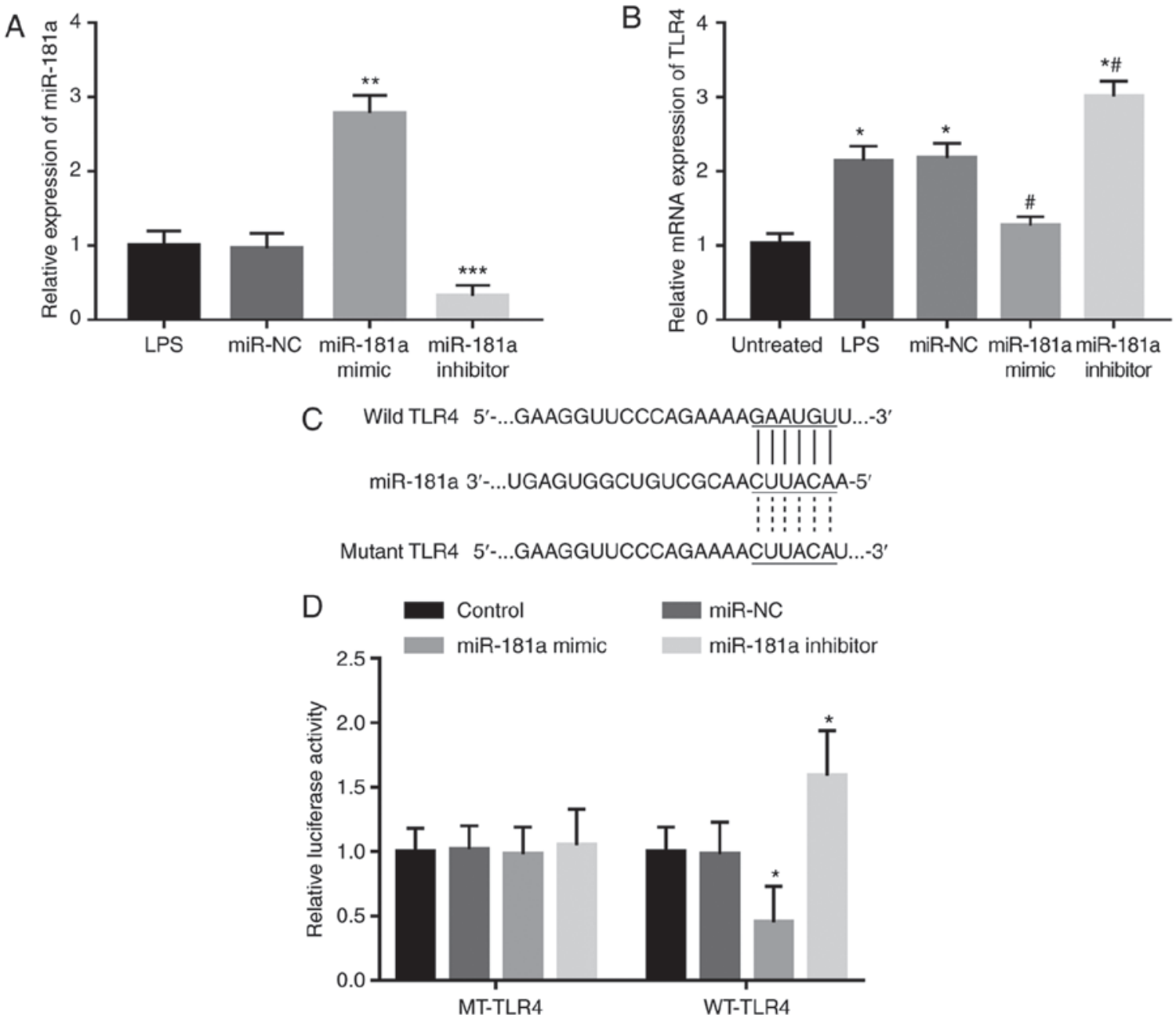

Figure 4. miR-181a directly inhibits the expression of TLR4 in primary monocytes. (A) The expression of miR-181a was increased by miR-181a mimics but was decreased by miR-181a inhibitor $\left({ }^{* *} \mathrm{P}<0.01,{ }^{* * *} \mathrm{P}<0.001 \mathrm{vs}\right.$. LPS). (B) The increase in TLR4 induced by LPS was suppressed by overexpression of miR-181a but was promoted by the reduction of miR-181a in the monocytes (" $\mathrm{P}<0.05$ vs. Untreated; ${ }^{\#} \mathrm{P}<0.05$ vs. LPS). (C) Complementary sequence of miR-181a in the 3'-UTR of TLR4. (D) Luciferase activity of the luciferase reporter plasmid carrying the WT 3'-UTR sequence of TLR4 was inhibited by overexpression of miR-181a, but was enhanced by inhibition of miR-181a ("P<0.05 vs. Control). miR, microRNA; LPS, lipopolysaccharide; MT, mutant; WT, wild-type; TLR, Toll-like receptor; NC, negative control; UTR, untranslated region.
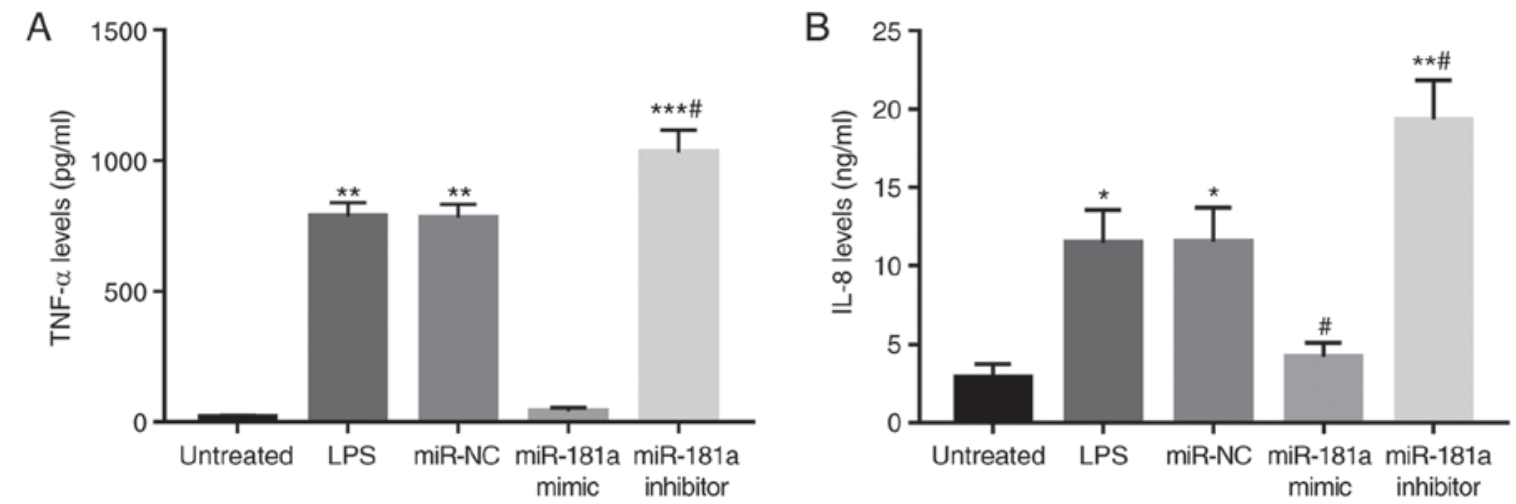

Figure 5. Effects of miR-181a on LPS-induced inflammation. LPS stimulation in the monocytes led to increased levels of (A) TNF- $\alpha$ and (B) IL-8, but these increases were suppressed by overexpression of miR-181a and were promoted by inhibition of miR-181a. ${ }^{*} \mathrm{P}<0.05,{ }^{* *} \mathrm{P}<0.01,{ }^{* * * *} \mathrm{P}<0.001$ vs. Untreated; ${ }^{*} \mathrm{P}<0.05$ vs. LPS. miR, microRNA; LPS, lipopolysaccharide; TNF, tumor necrosis factor; IL, interleukin; NC, negative control.

Thus, our study data may provide a diagnostic biomarker to screen the NS cases from the infection cohort. The expression analysis data shown a significant decrease in the expression of miR-181a in the serum specimens of patients with NS compared with the controls. In a study by Chen et al (16), downregulated expression of circulating miR-181a was also 
reported in patients with NS. Thus, miR-181a may have a pivotal role in the progression of NS.

Accurate diagnosis is the first and most important prerequisite of the efficient treatment of diseases. To improve the treatment of infectious diseases in neonates, it is of value to screen NS cases from the neonates with pneumonia/respiratory tract infection. CRP, WBC and PCT are the established and widely used diagnostic biomarkers for NS, but their application has limited specificity (27). Previous studies indicated that infection with bacteria, fungi or parasites may lead to increases in WBC and the levels of CRP and PCT (29). Thus, elevated WBC, CRP or PCT may be detected in certain infectious and inflammatory diseases other than NS, including pneumonia (30) and respiratory infection (31). In the present study, patients with NS and neonates with respiratory infection or pneumonia as the control group were enrolled. No significant differences were observed in age, sex and body weight between the two groups, but the cases with NS had significantly higher PCT levels than the controls. Regarding the CRP and WBC count, the difference did not reach statistical significance, although their values were increased in the NS group compared with that in the controls, which may be due to the increased inflammatory responses induced by respiratory infection in the control neonates. Thus, in addition to the detection of biological biomarkers, the clinical manifestations, as well as further verification from bacterial cultures, is essential for the diagnosis of NS.

miRNAs are considered ideal diagnostic tools for various human diseases, which is mainly due to their specific expression patterns and stability in blood samples (32). For instance, downregulated expression of miR-124 in the serum was previously described as a diagnostic biomarker for patients with osteosarcoma (33). Increased serum levels of miR-155-5p and miR-133a-3p were proven to serve as diagnostic and prognostic biomarkers in patients with sepsis (34). In patients with NS, upregulated expression of miR-15a/16 was also determined to be a diagnostic biomarker (18). Given the markedly decreased expression of miR-181a in the serum samples of patients with $\mathrm{NS}$, an ROC analysis was performed in the present study, demonstrating that aberrant expression of miR-181a may serve as a diagnostic biomarker to screen NS patients from the neonates with pneumonia/respiratory tract infection, with relatively high sensitivity and specificity. At present, the available diagnostic methods for NS are limited by their poor sensitivity or specificity. The present study may provide novel and efficient diagnostic biomarkers for patients with NS.

NS is characterized by inflammatory responses. TLR4 is a protein that has a key role in the innate immune system (35). TLR4 is sensitive to external signals and is an important mediator for inflammatory events (36). He et al (17) reported that TLR4 is a target gene of miR-181a in immune thrombocytopenia. Thus, the present study also focused on the effect of miR-181a on the expression of TLR4 in monocytes collected from the blood samples of NS patients. The results indicated that TLR4 was a target gene of miR-181a and was downregulated by overexpression of miR-181a. Furthermore, the role of miR-181a in the regulation of inflammatory cytokines was explored in monocytes treated with LPS. The LPS-induced increases in the levels of inflammatory cytokines were all suppressed following overexpression of
miR-181a, indicating the suppressive role of miR-181a in the regulation of LPS-induced inflammation. Collectively, it may be indicated that miR-181a may inhibit the LPS-induced inflammatory response by downregulation of TLR4. Although the present study provided evidence for the regulatory effect of miR-181a on the expression of TLR4, further research is required to confirm this interaction and investigate the effects of miR-181a on TLR4-associated signaling. Autophagy, which can be regulated by TLR4, has been reported to be involved in the progression of sepsis (37). Interestingly, previous studies have found a regulatory role for miR-181a on autophagy in the pathogenesis of some diseases, such as myocardial hypertrophy (38) and gastric cancer (39). Thus, it was deduced that the miR-181a/TLR4 axis might also be involved in the regulation of autophagy in NS development. However, this hypothesis was not investigated in the present study, which is one of the limitations of this present study. Additionally, the accuracy of the clinical research data may be limited by the small sample size and further investigations with larger research cohorts are required.

In conclusion, the present study revealed that the serum expression of miR-181a is downregulated in patients with NS and the dysregulation of miR-181a serves as a candidate diagnostic biomarker for NS. Overexpression of miR-181a in monocytes was able to improve the LPS-induced inflammatory reaction by targeting TLR4, which may further uncover the pathologic mechanisms of action underlying the development of NS.

\section{Acknowledgements}

Not applicable.

\section{Funding}

No funding was received.

\section{Availability of data and materials}

The datasets used and/or analyzed during the current study are available from the corresponding author on reasonable request.

\section{Authors' contributions}

GL made substantial contributions to the conception and design of the study, analysis and interpretation of data and revision of the manuscript. WL and JG were involved in the acquisition of data and drafting of the manuscript. All authors gave final approval of the version to be published.

\section{Ethics approval and consent to participate}

The experimental protocols were approved by the Ethics Committee of Yidu Central Hospital of Weifang (Weifang, China) and written informed consent was obtained from the families of the patients.

\section{Patient consent for publication}

Not applicable. 


\section{Competing interests}

The authors declare that they have no competing interests.

\section{References}

1. Gotts JE and Matthay MA: Sepsis: Pathophysiology and clinical management. BMJ 353: i1585, 2016.

2. Shane AL, Sanchez PJ and Stoll BJ: Neonatal sepsis. Lancet 390: 1770-1780, 2017.

3. Sharma D, Farahbakhsh N, Shastri S and Sharma P: Biomarkers for diagnosis of neonatal sepsis: A literature review. J Matern Fetal Neonatal Med 31: 1646-1659, 2018.

4. de Prost N, Razazi K and Brun-Buisson C: Unrevealing culture-negative severe sepsis. Crit Care 17: 1001, 2013.

5. Boskabadi $\mathrm{H}$ and Zakerihamidi M: Evaluate the diagnosis of neonatal sepsis by measuring interleukins: A systematic review. Pediatr Neonatol 59: 329-338, 2018.

6. Omran A, Maaroof A, Saleh MH and Abdelwahab A: Salivary C-reactive protein, mean platelet volume and neutrophil lymphocyte ratio as diagnostic markers for neonatal sepsis. J Pediatr (Rio J)94: 82-87, 2018.

7. Iroh Tam PY and Bendel CM: Diagnostics for neonatal sepsis: Current approaches and future directions. Pediatr Res 82: 574-583, 2017.

8. Shi GL, Chen Y, Sun Y, Yin YJ and Song CX: Significance of serum MicroRNAs in the auxiliary diagnosis of non-small cell lung cancer. Clin Lab 63: 133-140, 2017.

9. Cui S, Liu L, Wan T, Jiang L, Shi Y and Luo L: MiR-520b inhibits the development of glioma by directly targeting MBD2. Am J Cancer Res 7: 1528-1539, 2017.

10. Shen DW, Li YL, Hou YJ, Xu ZD, Li YZ and Chang JY: MicroRNA-543 promotes cell invasion and impedes apoptosis in pituitary adenoma via activating the $\mathrm{Wnt} / \beta$-catenin pathway by negative regulation of Smad7. Biosci Biotechnol Biochem 83 1034-1044, 2019.

11. Liu CH, Wang Z, Huang S, Sun Y and Chen J: MicroRNA-145 regulates pathological retinal angiogenesis by suppression of TMOD3. Mol Ther Nucleic Acids 16: 335-347, 2019.

12. Wang JK, Wang Z and Li G: MicroRNA-125 in immunity and cancer. Cancer Lett 10: 134-145, 2019.

13. Karam RA and Abd Elrahman DM: Differential expression of miR-155 and Let-7a in the plasma of childhood asthma: Potential biomarkers for diagnosis and severity. Clin Biochem 68: 30-36, 2019.

14. Qiu Z, Li H, Wang J and Sun C: miR-146a and miR-146b in the diagnosis and prognosis of papillary thyroid carcinoma. Oncol Rep 38: 2735-2740, 2017.

15. Qin C, Huang RY and Wang ZX: Potential role of miR-100 in cancer diagnosis, prognosis, and therapy. Tumour Biol 36: 1403-1409, 2015 .

16. Chen J, Jiang S, Cao Y and Yang Y: Altered miRNAs expression profiles and modulation of immune response genes and proteins during neonatal sepsis. J Clin Immunol 34: 340-348, 2014.

17. He YZ, Lu RF, Zhu C and Hua JY: Qian five rhinoceros gindeng (QFRG) protects against development of immune thrombocytopenia via miR-181a inhibition of TLR-4 expression. Int J Clin Exp Med 8: 6986-6993, 2015.

18. Wang X, Wang X, Liu X, Wang X, Xu J, Hou S, Zhang X and Ding Y: miR-15a/16 are upreuglated in the serum of neonatal sepsis patients and inhibit the LPS-induced inflammatory pathway. Int J Clin Exp Med 8: 5683-5690, 2015.

19. Subspecialty Group of Neonatology Pediatric Society Chinese Medical Association; Editorial Board Chinese Journal of Pediatrics: Protocol for diagnosis and treatment of neonatal septicemia. Zhonghua Er Ke Za Zhi 41: 897-899, 2003 (In Chinese).

20. Livak KJ and Schmittgen TD: Analysis of relative gene expression data using real-time quantitative PCR and the 2(-Delta Delta C(T)) method. Methods 25: 402-408, 2001
21. Yu HR, Chen RF, Hong KC, Bong CN, Lee WI, Kuo HC and Yang KD: IL-12-independent Th1 polarization in human mononuclear cells infected with varicella-zoster virus. Eur J Immunol 35: 3664-3672, 2005.

22. Huang G, Lou T, Pan J, Ye Z, Yin Z, Li L, Cheng W and Cao Z: MiR-204 reduces cisplatin resistance in non-small cell lung cancer through suppression of the caveolin-1/AKT/Bad pathway. Aging (Albany NY) 11: 2138-2150, 2019.

23. Oh YS, Bae GD, Park EY and Jun HS: MicroRNA-181c inhibits interleukin-6-mediated beta cell apoptosis by targeting TNF- $\alpha$ expression. Molecules 24: E1410, 2019.

24. Jiang D, Li M, Yu Y, Shi H and Chen R: MicroRNA-34a aggravates coxsackievirus B3-induced apoptosis of cardiomyocytes through the SIRT1-p53 pathway. J Med Virol 91: 1643-1651, 2019.

25. Ma Y, Liu Y, Hou H, Yao Y and Meng H: MiR-150 predicts survival in patients with sepsis and inhibits LPS-induced inflammatory factors and apoptosis by targeting NF- $\kappa \mathrm{B} 1$ in human umbilical vein endothelial cells. Biochem Biophys Res Commun 500: 828-837, 2018

26. Wang Z, Ruan Z, Mao Y, Dong W, Zhang Y, Yin N and Jiang L: miR-27a is up regulated and promotes inflammatory response in sepsis. Cell Immunol 290: 190-195, 2014.

27. Dhas BB, Dirisala VR and Bhat BV: Expression levels of candidate circulating microRNAs in early-onset neonatal sepsis compared with healthy newborns. Genomics Insights 11: 1178631018797079, 2018.

28. Cerone JB, Santos RP, Tristram D, Lamson DM, Stellrecht KA, St George K, Horgan MJ and Rios A: Incidence of respiratory viral infection in infants with respiratory symptoms evaluated for late-onset sepsis. J Perinatol 37: 922-926, 2017.

29. Yu Z, Zhang B, Xu Y, Hao Y, Tang J, Yu W and Gu Q: Analysis of clinical characteristics of bloodstream infection in patients with immune function inhibition. Zhonghua Wei Zhong Bing Ji Jiu Yi Xue 30: 1087-1090, 2018 (In Chinese).

30. Guo S, Mao X and Liang M: The moderate predictive value of serial serum CRP and PCT levels for the prognosis of hospitalized community-acquired pneumonia. Respir Res 19: 193, 2018

31. Kim HS, Won S, Lee EK, Chun YH, Yoon JS, Kim HH and Kim JT: Pentraxin 3 as a clinical marker in children with lower respiratory tract infection. Pediatr Pulmonol 51: 42-48, 2016.

32. Bertoli G, Cava $\mathrm{C}$ and Castiglioni I: The potential of miRNAs for diagnosis, treatment and monitoring of breast cancer. Scand J Clin Lab Invest Suppl 245: S34-S39, 2016.

33. Cong C, Wang W, Tian J, Gao T, Zheng W and Zhou C: Identification of serum miR-124 as a biomarker for diagnosis and prognosis in osteosarcoma. Cancer Biomark 21: 449-454, 2018.

34. Lan C, Shi X, Guo N, Pei H and Zhang H: Value of serum miR-155-5p and miR-133a-3p expression for the diagnosis and prognosis evaluation of sepsis. Zhonghua Wei Zhong Bing Ji Jiu Yi Xue 28: 694-698, 2016 (In Chinese)

35. Wang J, Wang R, Yang J, Yang X, Hu S, Wang H, Zhou C, Xiong W, Wen Q and Ma L: Glucocorticoids differentially regulate the innate immune responses of TLR4 and the cytosolic DNA sensing pathway. Int Immunopharmacol 47: 190-198, 2017.

36. Alexander CM, Xiong KN, Velmurugan K, Xiong J, Osgood RS and Bauer AK: Differential innate immune cell signatures and effects regulated by toll-like receptor 4 during murine lung tumor promotion. Exp Lung Res 42: 154-173, 2016.

37. Carchman EH, Whelan S, Loughran P, Mollen K, Stratamirovic S, Shiva S, Rosengart MR and Zuckerbraun BS: Experimental sepsis-induced mitochondrial biogenesis is dependent on autophagy, TLR4, and TLR9 signaling in liver. FASEB J 27: 4703-4711, 2013

38. Li AL, Lv JB and Gao L: MiR-181a mediates Ang II-induced myocardial hypertrophy by mediating autophagy. Eur Rev Med Pharmacol Sci 21: 5462-5470, 2017.

39. Zhao J, Nie Y, Wang H and Lin Y: MiR-181a suppresses autophagy and sensitizes gastric cancer cells to cisplatin. Gene 576: 828-833, 2016. 